\title{
Combination of Sacral Spinal Anesthesia and Popliteal Fossa Block on Surgical Side for Below Knee Surgery
}

\author{
Radhashyam Paria ${ }^{1}$, Smarajit Surroy ${ }^{1}$, Mousumi Majumder ${ }^{1}$, Baishakhi Paria ${ }^{2}$, \\ Soma Sengupta ${ }^{3}$, GoutamDas, ${ }^{4}$ Anshuman Paria ${ }^{5}$ \\ 1(dept of Anesthesiology, Howrah Orthopedic Hospital, Eastern Railways, WB) \\ 2(Department of Community Medicine, National Medical College) \\ 3(dept of Anesthesiology, Columbia Asia Hospital, Salt Lake, WB) \\ 4(Department of Pediatric Medicine, North Bengal Medical College, West Bengal) \\ 5(dept of Neonatology, SSKM hospital, WB)
}

\begin{abstract}
:
Background: The beneficial effects of two central neuraxial blocks in combined form are well acceptable and well tolerable by patients. Beneficial effects of both components are utilized, minimizing their drawbacks.

Aims: Our study is designed to evaluate the use of peripheral ( popliteal fossa block) and central nerve block (spinal) in combined form for below knee surgical procedures.

Methods: 30 patients of 60-90 years old with ASA physical status of classification II and III were selected for below knee surgical procedures undergoing popliteal fossa block 20 minutes before sacral spinal anesthesia. The popliteal fossa block was achieved with $30 \mathrm{ml}$ of o.375\% injection bupivacaine through lateral approach at the level of upper broader of patella with help of nerve stimulator needle. Sacral spinal anesthesia was introduced through second dorsal foramen of sacrum by intrathecal administration of three ml of $0.5 \%$ injection bupivacaine.

Results: The hemodynamic stability along with profound and prolonged analgesia before, during and after surgical procedure is the most interesting characteristic feature of this study. Preoperative popliteal fossa block induces analgesic effect at the fracture site and contributes comforts to patients at the time of positioning the patient for administration of sacral spinal anesthesia. It acts as an adjuvant to spinal anesthesia like intrathecal administration of fentanyl or clonidine with the central depression.

Conclusion: It provides analgesia before spinal as preoperative medication without central depression, cardiovascular stability during surgical procedures under spinal anesthesia and prolonged analgesia after surgery.

Keywords: sacral spinal; popliteal fossa; below knee surgery; combined block
\end{abstract}

\section{Introduction:}

The predictability of peripheral nerve block (PNB) in the relation to providing profound analgesia to the blocked area is worldwide acceptable without cardiovascular fluctuation. The central neuraxial block like sacral spinal anesthesia (SA) produces analgesia of the larger part of the body with intrathecal injection of relatively small amount of local anesthetic. But its unpredictability in connection with intrathecal spread of local anesthetic drug through cerebrospinal fluid (CSF) leads to fluctuation of block height resulting in either adequate or inadequate block for projected surgery. The extensive intrathecal spread of local anesthetic in cephalad direction may be responsible for grave complication like total spinal or cardiovascular instability. Over and above, spinal anesthesia is unable to abolish completely neural transmission in block area. The raised sensory threshold, produced in blocked area by combined spinal epidural anesthesia (CSE) is found to be more than that found in the area blocked by spinal anesthesia.

It is also found that CSE produces physiologically denser block than either of two techniques alone. With above stand point, it is also clear to accept the view that administration of the high-quality analgesia immediate after operation is an essential part to control surgical stress response to major surgeries. [2] The analgesia, contributed by spinal anesthesia at the end of the operation, may not be sufficient enough to fulfill the above purpose. However, the combined anesthesia of spinal and preoperative specific peripheral nerve block offers the advantages of both components by minimizing their respective disadvantages, resulting in hemodynamic stability, rapid onset of block and profound, prolonged analgesia during and after surgery. We hypothesized to evaluate the combined effects of preoperative specific peripheral nerve block (PNB) and sacral spinal anesthesia (SA). 


\section{Methods:}

With the acceptance of written informed consent from all participants of the study and procedure, we recruited 30 patients of ASA physical status classification 11 and 111 for lower limbs surgeries (tibia) in the period of January, 2014 to July, 2014.

With preoperative assessment of all patients to exclude the contraindications of specific PNB and SA anesthesia in the sacral area, we started intravenous infusion with dextrose in normal saline in the operation theater with noninvasive monitoring of blood pressure, heart rate, oxygen saturation and electrocardiography.

Two divisions of the sciatic nerve namely tibial and common peroneal are usually blocked through the method of the lateral approach between tendons of vastus lateralis and biceps femoris in the supine position of patients.[ We identified the anatomical landmarks like lateral epicondyle and groove between of vastus lateralis and biceps femoris. Insertion point was located at $7 \mathrm{~cm}$ proximal from lateral femoral condyle in the groove between above two muscles. The nerve stimulator needle was inserted perpendicularly to the skin in a horizontal plane, with initial current of $0.8 \mathrm{~mA}$ at the located point. The nerve stimulator needle was advanced to touch the femur. After contact, the needle was withdrawn and redirected 30 degrees posteriorly to have stimulation of nerves. Stimulation of tibial nerve resulted in plantar flexion and inversion. Stimulation of common peroneal resulted in dorsiflexion and eversion. In some cases, nerves were localized, but it was not possible in all cases. In that case, needle was withdrawn to the skin surface and reinserted further changing the angle of 5-10 degree either in an anterior direction or posterior direction. When appropriate stimulation was identified, $30 \mathrm{ml}$ of $0.375 \%$ of injection bupivacaine was injected after aspiration to exclude the intravascular placement of the needle.

After completion of popliteal fossa block, anatomical landmarks of dorsal foramens of sacrum were identified. We identified the spinous process of fourth lumbar vertebra (L4) and tip of coccyx. Next, we located the mid-point of above two landmarks as third sacral vertebra (S3). The first sacral vertebra (S1) was located as mid-point of L4 and S3. The second sacral vertebra (S2) was found at the mid-point between S1 and S3. The spinous processes of $\mathrm{S} 1$ and $\mathrm{S} 2$ are found approximately $2.5 \mathrm{~cm}$ apart, and their respective foramina lie $1.5 \mathrm{~cm}$ caudal and lateral to them. Similarly S3 foramina lie $1.5 \mathrm{~cm}$ caudal and lateral to S3 vertebra.

The above described land marks were further confirmed by visualizing their relations to other structures. L4 is on the line joining the iliac crests. The tip of coccyx lies on the line joining the femoral acetabula. The spinous process of S2 lies at the level of posterior superior iliac spine. It is often detected by a dimple of skin. The S3 lie at the level of posterior inferior iliac spine.

We infiltrated the identified area with $6 \mathrm{ml}$ of $1 \%$ xylocaine and administered sacral spinal anesthesia[3,4,5] through $2 \mathrm{nd}$ dorsal foramen of sacrum with intrathecal injection of $3 \mathrm{ml}$ of $0.5 \%$ injection bupivacaine. The intrathecal injection was administered after noticing clear flow of cerebrospinal fluid [CSF] to avoid intravascular injection.

We estimated the onset of sensory or motor block(interval between spinal and loss of sensation or motor function of lower limbs respectively) and duration of sensory or motor block(interval between spinal and recovery of sensation or motor respectively) and presented all collected data for statistical analysis.

\section{Results:}

All participants of combined central and peripheral nerve block procedure showed hemodynamic stability by minimizing the drawbacks of central neuraxial block like intrathecal procedure. Preoperative administration of sciatic nerve block at the level of the popliteal fossa offered greater quality of analgesia along its distribution below the applied site (knee). This kind of analgesia was sufficient to relieve the preoperative pain at the fracture site and made the patients cooperative at the time of administration of sacral spinal anesthesia through the second dorsal foramen of sacrum. No additive was needed in peri-operative period for prolongation of block or to supply the extra analgesia. The cephalic extension of sensory block was limited to tenth thoracic dermatome after the end of six minutes of administration of the sacral spinal anesthesia. Assessment of motor block, estimated by modified Bromage scale was revealed the result of complete motor block scoring 3. The central and specific peripheral nerve block, developed to repair the tibial fracture showed prolonged period of analgesia in the postoperative period [15 hr ]. The results of the demographic profile, hemodynamic profile and nerve block profile are given in separate table [table no 1].

\section{Discussion:}

Regional anesthesia is not "one size its all" anesthesia. It is the combination of selective peripheral nerve block techniques of anesthesia that can produce anesthesia and postoperative analgesia as selective as a surgical procedure itself. The technological advancement in connection of nerve stimulation leads to rapid growth in both number and types of peripheral nerve block techniques. The combination of sacral spinal anesthesia and popliteal fossa block produces anesthesia with profound analgesia for below knee surgeries. 
The popliteal block is a block of the sciatic nerve at the level of the popliteal fossa after its division. The sciatic nerve is a nerve bundle of two separate nerve trunks. They are the tibial and the common peroneal nerves. These two nerve bundles are enveloped by a common epineural sheath unto its division at the level of the popliteal fossa. In this fossa, it divides into two components as the tibial and the common peroneal nerves.

This division of the sciatic nerve usually takes place at the level of 4 to $10 \mathrm{~cm}$ proximal to the popliteal fossa crease. After the division of the sciatic nerve, the common peroneal nerve travels downward and laterally, descending along the head and neck of the fibula. Its terminal branches are the superficial and deep peroneal nerves. The tibial nerve is the larger of the two divisions of the sciatic nerve. It continues its path vertically through the popliteal fossa, and its terminal branches are the medial and lateral plantar nerves. In the popliteal fossa, the sciatic is situated lateral and superficial to popliteal an artery. This relationship is practically important to insert nerve stimulator needle or to avoid the arterial puncture and intravascular administration of local anesthetic. The sciatic nerve and its terminal divisions are covered by separate perineural sheath and they are not entangled in the neurovascular bundle. This separate anatomical position of vascular and neural components explain the relatively low risk of systemic toxicity and vascular punctures with a popliteal block. However, this anatomical arrangement of vital structures like artery, vein and nerve can not rule out the possibility of intravascular needle placement. For the avoidance of such potential danger, frequent careful aspiration and meticulously slow injection are most essential.

The PNB is a predictable process of nerve block to provide profound analgesia to the blocked area supplied by blocked nerve without cardiovascular disturbance although it needs a long time to develop with the large quantity of local anesthetic. The expected long time of initiation of block can be shortened by combined use of intrathecal block (SA) with PNB for projected surgery of lower limbs. The intentional use of PNB and SA in combined form contributes beneficial effects of both components by minimizing their respective disadvantages. Spinal component contributes rapid onset of action whereas PNB component offers prolongation of action acting as adjutants to SA. The peripheral nerve block provides the profound analgesia without central depression.

Popliteal fossa block is demonstrated to block the two divisions of the sciatic nerve in the popliteal fossa with practical evidence of dorsiflexion with eversion and plantar flexion with inversion produced by stimulation of common peroneal and tibial nerve respectively. This technique of knee approach is highly appropriate and effective. [6,7\}

The preoperative administration of popliteal fossa block in a supine position of patients with minimal movement of surgical site, offers more comfort while positioning the patient for administration of sacral spinal anesthesia. This PNB also delivers high quality of analgesia during and after surgery without depression of higher function. On the other hand, this PNB helps to maintain hemodynamic stability that is seemed to be disturbed by the subarachnoid anesthesia.

Dramatic reduction of pain when applied before surgery helps the patient to sit on the operation table with comfort for intrathecal procedure. During the surgical procedure, it acts as adjutant to spinal anesthesia potentiating analgesic effect to long time. This potentiating analgesic effect extends to post operative period up to $15-18$ hours. [8,9] Lateral approach $[10,11,12]$ is useful and appropriate in fracture cases owing to less movement during the procedure of popliteal fossa block.

Spinal anesthesia is generally considered as most reliable method of regional anesthesia. The insertion of the spinal needle is usually straightforward, appearance of clear cerebrospinal fluid (CSF) is the evidence of perfect placement of the spinal needle in subarachnoid space and deposition of local anesthetic within CSF confirms the intrathecal spread. But frequently, such beneficial regional block is associated with cardiovascular instability. Sometimes, extensive intrathecal spread may complicate this regional block with total spinal. The predictability of the spinal anesthesia depends on intrathecal spread of local anesthetic both in extending and degree. Adequate spread leads to adequate block height for proposed surgery whereas inadequate intrathecal spread of local anesthetic leads to inadequate block for projected surgery. Overall fixed duration of anesthesia,

Spinal or epidural neuraxial block is not able to abolish completely neural transmission in block area. The raised sensory threshold, produced in blocked area by combined spinal epidural anesthesia (CSE) is found to be more than that found in the area blocked by SA or EA alone1. Thus, it is found that CSE produces physiologically denser block than either of two techniques alone. High quality analgesia immediate after operation is the essential part to control surgical stress response to major surgeries.2 Persistence of pain sensation and badly controlled surgical stress response are frequently associated with spinal or epidural form of anesthesia when they are used alone, but are absent in combined spinal epidural form of anesthesia when they are used joint together.

The most unfavorable and undesirable fixed duration of anesthesia, contributed by intrathecal procedure needs adjutants like opioid [14 ] or clonidine [15-17 ] or fentanyl [18-21 ] or morphine [22-25] for prolongation of action, although this attempt is complicated with the central depression and postoperative hypoxia particularly in elderly patients. 
The combined spinal epidural form of anesthesia that offers the advantages of both components by decreasing their respective disadvantages, is the best technique for prolonged operation with provision of postoperative analgesia. Spinal component contributes rapid onset of action whereas epidural component offers prolongation of action. This sophisticated form of anesthesia can supply immediate postoperative analgesia without central depressive effects and systemic analgesia although it needs specialization and service of expert hands.

The sacral spinal anesthesia, administered through the second dorsal foramen of sacrum to deposit the local anesthetic at the bottom of the dural sac, acquires tendency to block T12 dermatomes (12th thoracic ). The sacral spinal anesthesia possesses an unlimited importance as segmental block of lumbosacral nerve roots of spinal cord. This segmental involvement is highly acceptable for the purpose of lower limbs surgeries with prolonged postoperative analgesia and cardiovascular stability. Such beneficial effects of lumbosacral segmental block are further enforced by addition of analgesic activities during and after operation without central depressive effects like hypoxia seen after operation.

\section{Conclusion:}

With hemodynamic stability and prolonged analgesic effects during and after operation without central depression, combination of sacral spinal anesthesia and popliteal fossa block on the surgical side is appropriate for lower limb surgery on tibia and foot.

\section{References}

[1]. Dirkes WE, Rosenberg J, Lund C, Kehlet H. The effect of subarachnoid lidocaine and combined subarachnoid lidocaine and epidural lidocaine on electrical sensory thresholds. Reg Anesth $1991 ; 16: 262-4$

[2]. Mcleod GA, Dell K, Smith C, Wildsmith J.A.W. Measuring the quality of epidural block for abdominal surgery. Br J Anesth 2006; 96:633-9.

[3]. Paria R, Surroy S, Majumder M, Paria A, Paria B, Das G. Sacral spinal anesthesia. Indian J Anesth. 2014; 58(1) : 80-2

[4]. Paria R, Surroy S, Majumder M, Paria B, Sengupta S, Paria A. Sacral Saddle Block IOSR-JDMS 2014; 13(4) 39-40.

[5]. Paria R, Surroy S, Majumder M, Paria B, Sengupta S, Paria A. Sacral Epidural Anesthesia IOSR-JD MS 2014; 13(5) 10-11

[6]. Hansen,E, Eshelman,M.R, Cracchiolo, A.3rd . Popliteal fossa neural blockade as the sole anesthetic technique for outpatient foot and ankle surgery. Foot Ankle Int.21:38, 2000.

[7]. Singelyn, F. J, Gouverneur, J. M., Gribomont, B. F. Popliteal sciatic nerve block aided by nerve stimulator: A reliable technique for foot and ankle surgery. Reg. Anesth. 16: 278, 1991.

[8]. Mcleod, D, H., Wong, D.H., Vaghadia, H., Claridge, R.J., Merrick,P.M., Lateral popliteal sciatic nerve block comparedwith ankle block for analgesia following foot surgery. Can. J. Anesth. 42: 765. 1995

[9]. Zetlaoui,PJ, Bouaziz,H. Lateral approach to the sciatic nerve in the popliteal fossa Anesth Analg. 1998;87;79

[10]. Vloka,J,D.,Hadzic, A,Kitain,E.,Lesser, J.B., Kuroda,M.,April,E.W., Thys, D.M., Aconsiderations for sciaticnerve block in the popliteal fossathroughthe lateral approach. Reg.Anesth.21;414,1996.

[11]. Paqueron,X,.Bouaziz,H.,Macalou,D.,LabailleT.,Merle,M.,Laxenaire,M.C.,Benhamon,D., T approach to the sciatic nerve at the popliteal fossa: One or two injections? Anesth. Analg.89;1221,1999.

[12]. Hadzic A, Vloka JD: A comparison of the posterior versus lateral approaches to the block of the sciatic nerve in the popliteal fossa. Anesthesiology 1998; 88; 14

[13]. Vloka JD, Hadzic A, April E, Thys DM. The division of the sciatic nerve popliteal fossa: anatomical implications for popliteal nerve block. Anesthesia \& Analgesia 2001;92:215-7

[14]. Popping DM, Elia N, Marret E, Wenk M and Tramer MR. Opioids added to local anesthetics for single-shot intrathecal anesthesia in patients undergoing minor surgery: a meta-analysis of randomized trials. Pain.2012; 153:784-93.

[15]. Sethi BS, Samuel M, Sreevastava D. Efficacy of analgesic effects of low dose intrathecal clonidine as adjuvant to bupivacaine. Indian J Anaesth 2007;51:415-9.

[16]. Grandhe RP, Wig J, Yaddanapudi LN. Evaluation of bupivacaine-clonidine combination for unilateral spinal anesthesia in lower limb orthopaedic surgery. J Anaesth Clin Pharmacol 2008;24:155-8.

[17]. Niemi L. Effects of intrathecal clonidine on duration of bupivacaine spinal anaesthesia, hemodynamic and postoperative analge sia in patients undergoing knee arthroscopy. Acta Anesthesiol Scand 1994;38:724-8.

[18]. Dahlgren G, Hultstrand C, Jakobsson J et al. Intrathecal sufentanil, fentanyl or plaecebo added to bupivacaine for caesarean section. Anesth Analg 1997; 85; 1288-93.

[19]. Manullang TR, Viscomi CM, Pace NL. Intrathecal fentanyl is superior to intravenous ondansetron for prevention of perioperative nausea during caesarean delivery with spinal anaesthesia. Anesth Analg 2000; 90: 1162-6.

[20]. Shendi D, Cooper GM, Bowden MI. The influence of IT fentanyl on the characteristics of subarchnoid block for Caesarean section. Anaesthesia 1998; Jul 53(7): 706-10.

[21]. Chu CC, Shu SS, Lin SM et al. The effect of intrathecal bupivacaine with combined fentanyl in caesarean section. Acta Anaesthesiol Sin 1995; Sep 33(3): 149-54

[22]. Bailey PL, Rhondeau S, Schafer PG, et al Dose-response pharmacology of intrathecal morphine in human volunteers. Anesthesiology 1993;79:49-59.discussion 25A.

[23]. Beaussier M, Weickmans H, Parc Y, et al Postoperative analgesia and recovery course after major colorectal surgery in elderly patients: a randomized comparison between intrathecal morphine and intravenous PCA morphine. Reg Anesth Pain Med2006;31:531-8.

[24]. Blay M, Orban JC, Rami L, et al Efficacy of low-dose intrathecal morphine for postoperative analgesia after abdominal aortic surgery: a double-blind randomized study. Reg Anesth Pain Med 2006;31:127-33

[25]. Block BM, Liu SS, Rowlingson AJ, Cowan AR, Cowan JA Jr, Wu CL Efficacy of postoperative epidural analgesia: a metaanalysis. J Am Med Assoc2003;290:2455-63. 
Combination of Sacral Spinal Anesthesia and Popliteal Fossa Block on Surgical Side for Below Knee

\begin{tabular}{|l|l|}
\hline Table-1 Showing demographic profile, hemodynamic profile and analgesic profile of patients. \\
\hline DEMOGRAPHIC PROFILE & \\
\hline Age (years) & $78.38 \pm 8.08$ \\
\hline Height (Cm) & $28: 22$ \\
\hline Weight (Kg) & $58.04 \pm 3.02$ \\
\hline Sex (M:F) & $158.96 \pm 4.67$ \\
\hline HEMODYNAMIC PROFILE & \\
\hline Systolic blood pressure (mmHg) & $89.92 \pm 12.75$ \\
\hline Heart beats (beats/min) & $98.94 \pm 0.89$ \\
\hline Oxygen saturation (\%) & $112.32 \pm 7.62$ \\
\hline ANALGESIC PROFILE. & \\
\hline Postoperative analgesia (hours) & $15.82 \pm 2.34$ \\
\hline
\end{tabular}

